\title{
Teaching crystallography and X-ray diffraction online with MS Teams - experience
}

\author{
Radomír Kužel \\ Faculty of Mathematics and Physics, Charles University, Ke Karlovu 5, 12116 Praha 2 \\ kuzel@karlov.mff.cuni.cz
}

Many months of this pandemic brought high concentration on online teaching in basically all levels of education. Of course, the least problematic is such teaching in universities where many things can be transferred to online form without significant losses and in certain cases even with some benefits. Of course, not for the work that should teach students some manual skills. Otherwise, there are no limits for interactive communication during the online teaching. However, it may be easier for the teachers rather than for students who must sit at the computer many hours a day. Universities are supporting different platforms for online teaching. While for organizing of meetings I prefer to use Zoom or similar platforms, for teaching I have decided to prepare everything in MS Teams in the form for education where it is easy to create a team for the subject and assign students from the list of university students.

Our faculty required that all the presentations be recorded, and the records are available, in addition to presentations (ppt, pdf), to all relevant students till the end of semester. Some shared files like Excel or Word ones have possibility of multiple access of teacher and student. Probably the most useful part is Notebook that can contain different folders owned by teacher only, shared for all and owned by each individual student, respectively. In the shared folder, anybody can write formatted text, draw, insert pictures, tables directly in Teams or in One Note application with a few more advanced features. Students cannot see folders and pages of other students while the teacher can see everything. So, the teacher can easily click on the corresponding place of any student any time and see up-to-date information, e.g. where the student is during his/her task. Teacher can also write or draw directly to their documents. Usually, it is working quite quickly if the Internet is not too slow. The system was used for online teaching of fundamentals of crystallography and X-ray diffraction for smaller groups of students up to 10. In addition to simple examples and tests, graphical possibilities were used either with mouse or graphical tablet. The students had different symmetrical periodical 2D patterns with a task to draw elementary cell, corresponding symmetry elements, and determine the plane group from the list. In order, to make their life easier, they could use a portfolio of all symbols and it was then sufficient to move specific symbols to relevant positions. A similar way was used for space groups (complete diagrams of general positions with symmetry elements or vice versa complete diagrams of symmetry elements with general positions, the determination or estimation of the space group). The work was quite smooth.

A little more complicated was the preparation of online practical courses when the entrance of students to the faculty building was completely forbidden. One was the basic problem of powder diffraction - determination of lattice parameter of unknown cubic phase and then also phase analysis of mixture of 3-6 phases. This practical part always begins with a short excursion in X-ray laboratory showing them a few instruments, description of powder diffractometer, preparation of different samples, specimen alignment and automatic measurement in symmetrical scan. So, everything was recorded to videos and what was only missing for students was their own specimen preparation. This is followed by demonstration of fast evaluation of powder pattern and generation of a file with peak parameters. The students used the free program Winplotr. A short video tutorial how to use it quickly for simple fitting of XRD peaks was provided. Students used this output (each with different dataset) to index peaks according to procedure described on web link and determined the lattice parameter considering the instrumental aberrations. This was done in Excel file simultaneously accessible also by the teacher. The first part was closed by looking into the ICDD Powder Diffraction File and trial to find the phase (demo by the teacher). Usually, it was not found because the lattice parameter deviated from the database value from some reason that was discussed. Then the pattern of a mixture of phases was evaluated in commercial software (demo by the teacher), the list of peaks was generated $(2 \theta, \mathrm{d}, \mathrm{I})$ and the students obtained scanned education edition (ICDD material) of Hanawalt index and made the search "manually", again with different datasets. Interaction of the teacher was necessary. Finally, for homework, the students should download 30-days trial of program Match and use it for the phase analysis of the mixture (again a short video tutorial provided. More online "practical" tasks were prepared, for example study of textures and stresses in thin films showing different diffraction geometries and scans.

Real examinations could be realized after the winter semester in February 2021. In general, I have never heard so well-structured and correct answers. I think that the reasons were the following. Students had everything available in their Teams folders. Each student had to go through all the tasks and materials independently but except the direct online teaching in any time that was suitable for him/her, and I did the same. During the online teaching of these "practical" tasks direct presence of students was required but, for a few students, it was realized offline in a few steps according to the guidelines. This required more time (days, weeks) but finally it was always completed successfully. The students could return to some parts of presentations and if something were not clear, they could look at corresponding video part. So, my overall experience was positive. However, it should be noted that - the courses were not for larger groups of students, these tasks do not require any manual skills and can be then adopted quite easily for online form.

Keywords: online teaching, MS Teams

Acta Cryst. (2021), A77, C677 